\title{
Understanding Challenges and Opportunities of Preventive Blood Pressure Self-Monitoring at Home
}

\author{
Erik Grönvall \\ Department of Computer Science \\ Aarhus University \\ Aabogade 34, 8200 Aarhus, Denmark \\ gronvall@cs.au.dk
}

\author{
Nervo Verdezoto \\ Department of Computer Science \\ Aarhus University \\ Aabogade 34, 8200 Aarhus, Denmark \\ nervo@cs.au.dk
}

\begin{abstract}
The herein presented study explores socio-technical complexities that may occur when introducing preventive health-measurement technologies in older adults' daily routines and everyday lives. Using mainly blood pressure measurements in private homes, the study applied qualitative and quantitative methods to understand existing challenges and uncover opportunities of self-monitoring technologies to support preventive healthcare activities among older adults. Emerging challenges from our study were: rule complexity for self-measuring, reliability of measurements, interpretation, understanding and health awareness, the sharing activity for prevention, motivational sources, the role of the doctor for prevention, older adult's use of technology, the home as a distributed information space, and visualization of health data. An awareness of these challenges can help designers to develop better tools to support people's preventive self-monitoring needs compared with existing solutions. Furthermore, supporting the active and informed citizen can improve older adult's care abilities, awareness and activation towards preventive care.
\end{abstract}

\section{Author Keywords}

Preventive care; self-monitoring; home-based technology.

\section{ACM Classification Keywords}

H.5.m [Information Interfaces and Presentation ]:

Miscellaneous.

\section{General Terms}

Human Factors; Design; Management.

\section{INTRODUCTION}

The Danish social welfare state provides equal access to health care services for everyone. However, demographic changes challenge the Danish healthcare system as the population gets older and consequently age-related illnesses and chronic diseases follow. One example of an increasing age-related illnesses among older adults is high blood pressure (BP) [37]. For a general adult, high $\mathrm{BP}$ or hypertension is defined as a condition in which the systolic blood pressure (SBP - blood pressure when the heart is beating) is equal to, or greater than, $140 \mathrm{mmHg}$ and/or a diastolic blood pressure (DBP - blood pressure when the heart is relaxed) is equal to, or greater than, $90 \mathrm{mmHg}$ [29]. In Denmark, high BP Permission to make digital or hard copies of all or part of this work for personal or classroom use is granted without fee provided that copies are not made or distributed for profit or commercial advantage and that copies bear this notice and the full citation on the first page. Copyrights for components of this work owned by others than ACM must be honored. Abstracting with credit is permitted. To copy otherwise, or republish, to post on servers or to redistribute to lists, requires prio specific permission and/or a fee. Request permissions from Permissions@acm.org. ECCE' '13, August $26-28,2013$, Toulouse, France Copyright 2013 ACM 978-1-4503-2251-5/13/08 ...\$15.00. http://dx.doi.org/10.1145/2501907.2501962 is placed in the top five list of health risk factors [16] and according to the World Health Organization (WHO), high BP increases the risk of heart diseases and stroke [37]. WHO also defines high $\mathrm{BP}$ as one of the most prominent health risk factors in developed countries (among others such as obesity, high cholesterol, and tobacco consumption) [37]. In Denmark, $16 \%$ of the men and $19 \%$ of the women have severe hypertension, and high BP has reduced the life expectancy of Danes by 9 to 6 months (numbers from 2007) [16]. To address challenges related to an increasing older population, such as high BP, there is a neoliberalistic idealism to motivate Danish citizens to take responsibility of their own lives and healthcare management [27].

Institutional care is more resource-demanding compared with home-based care [11] and for people over 60 years old, moving from an independent to a dependent life situation is a major concern [19]. As a consequence, enabling citizens to be selfsufficient and sustain an independent lifestyle is one preferred strategy to reduce health care costs [5]. Shifting from a disease to a (proactive) individual health management promotes both homebased and preventive healthcare in society [4, 34]. Home-based self-monitoring technologies can be one way to support a continuous independent lifestyle among for example older adults. Home-based self-monitoring of diverse health parameters can also limit the 'white coat'-phenomenon [29]. The 'white-coat'-effect occurs for example when a person shows higher BP values at the hospital due to anxiety of being in a clinical environment, or vice versa (normal readings at the hospital and high values at home). This phenomenon has been identified to be particular evident in the older population, together with specific BP conditions (i.e. isolated systolic hypertension, hypotension, postural and postprandial hypotension, and pseudo hypertension) [29]. The variability of BP and its importance [39] for older adults makes $\mathrm{BP}$ a suitable candidate for studying self-monitoring technologies.

The purpose of this study is to explore socio-technical challenges of using preventive self-monitoring technologies. Through a research and innovation consortium [23], we have investigated the role of home-based preventive self-monitoring technologies with a focus on BP monitoring. The project served to a) gain a better understanding of preventive self-monitoring activities done by Danish citizens (e.g. older adults), b) collect people's concerns and attitudes regarding preventive self-monitoring technologies, c) get an overview of what challenges people have when performing preventive self-monitoring activities, and d) explore and uncover opportunities to facilitate preventive self-monitoring activities.

In this paper, we present challenges that emerged from our BP study related to: the rule complexity for self-measuring, reliability of measurements, interpretation, understanding and health awareness, the sharing activity for prevention, motivational 
factors, the role of the doctor for prevention, older adult's use of technology, the home as a distributed information space, and visualization of health data. Then, we propose a set of design opportunities for preventive self-monitoring technologies that can facilitate their integration into older adults' homes and everyday activities.

Before introducing our user studies, we will discuss related research focusing on older adults, non-clinical care management, and preventive self-monitoring technologies.

\section{RELATED WORK}

This section describes the aging process, the concept of preventive care, and introduces preventive self-monitoring technologies.

\section{The aging process}

The World Health Organization (WHO) states that there is a necessity to "reinvent our assumptions of old age" to promote healthy living [38]. As suggested by Fisk et al. [10], we acknowledge aging as a process within three dimensions (biological, psychological, and social) and that age is also taskcontext dependent. Fisk et al. also state that chronological age is suitable only as a marker for behavioral changes. Furthermore, Rockwood et al. [31] state that older adults in a clinical setting have different needs regarding the use of healthcare services. Rockwood et al. redefined the concept of frailty as a dynamic model in which the balance between assets (e.g. health, attitudes, resources) and deficits (e.g. illness, disability, dependence) can help to determine people's independence level. Thus, an unstable balance between assets and threating deficits can be used to identify frail older adults [31]. According to their model, a 50 years old adult who might have experienced a severe deterioration of function (biological, psychological, or social) due to for example cancer might fall in the group between healthy and frail older adults. As older adults can experience different healthcare needs [26, 31, 33], special attention should be given to older adults who fall between the two groups (healthy-frail) as they most likely can benefit from preventive care activities [33].

\section{Preventive care}

Mallery et al. [26] define prevention (in a healthcare context) as any action that "delays disease or slows its progression". Preventive health measures include primary, secondary and tertiary interventions [33]. Primary prevention focuses on decreasing the risk of an illness to appear (i.e. immunizations and health promotion). Secondary prevention focuses on early detection and treatment, especially for asymptomatic diseases (e.g. cancer, hypertension and dementia). Tertiary prevention focuses on rehabilitation to slow deterioration and decrease disability of symptomatic but unreported illnesses by using a comprehensive assessment of function (i.e. physical, psychological and social) $[18,26,33]$.

Kane et al. [18] present four main areas of interventions for preventive strategies: 1) specific conditions or illnesses that result in dysfunction (e.g. cardiovascular diseases, cancer), 2) risk factors that can be categorized as unhealthy behaviors rather than illnesses (e.g. smoking, lack of exercise, unhealthy diet), 3) conditions that require caregivers' interventions (e.g. depression, drug abuse, visual and hearing impairment), and 4) dealing with consequences of caregiving and medical care (e.g. drug side effects, function disability of overprotective environments) [18] Furthermore, it is important to understand the differences and similarities between prevention and promotion in healthcare [6]. Many health promotion measures (e.g. for older adults) are related to primary or tertiary prevention and aim for behavioral change in older adults at risk or to improve their functional capacity $[6,26]$. However, lifestyle changes or preventive care activities (e.g. healthy diet, regular exercise, avoiding alcohol or smoking) might be sufficient for some, but not all, healthy older adults. As older adults are not a homogeneous group, preventive care that aims to modify a risk factor after its effects has been identified might not be effective, especially for frail older adults [18]. An older adult with a diagnosed symptom may need monitoring and an individual assessment, including medication adherence-support, to control a specific condition (e.g. high BP [37], diastolic dysfunction [36]) and avoid excessive or insignificant treatment [29]. For example are people over 80 propense to diastolic issues (a lack of arterial-blood oxygen when the heart is relaxed) [36].

Furthermore, similarities and differences between chronic-illness management and prevention do exist and have been analyzed by Glasgow et al. [13]. They also investigated whether or not the chronic care model (CCM) proposed by Wagner [35] is suitable for prevention. Similarities include: a) ongoing planned care with "proactive follow-up"; b) complexity due to multiple health behavior changes or risk factors; c) illness/reactive care is inadequate; d) active patient involvement is required; e) patient activation tailoring shared decision making with physicians is important. Differences include: a) visits to the doctor for prevention are less frequent than for chronic illnesses; b) patients with chronic illnesses have stronger motivation to change health behaviors; c) patients' care needs are greater for chronic illness care (e.g. medication adherence [7]); d) prevention often happens outside the medical culture; and e) prevention is less often tracked [13]. Although differences and similarities do exist, Glasgow et al. [13] suggest that an extended version of CCM can be suitable to understand, improve and develop preventive care. Our study recognized these similarities and differences to better understand preventive self-monitoring practices at home.

\section{Preventive self-monitoring technology}

In comparison with disease management technology, preventive healthcare technologies have been less studied [4]. However, examples do exists such as Beaudine et al. [3] that use mockups of diverse visualizations to reveal user reactions to home monitoring for proactive health and wellbeing. One of the most common examples about prevention is accident detection (e.g. falls) that has been addressed using sensors and algorithms [9]. Furthermore, self-monitoring of health parameters, as part of primary prevention, has been tightly coupled with health promotion and challenges related to lifestyle and behavior changes [20]. In secondary prevention, the concept of embedded assessment have been introduced by Morris et al. [28] to promote the early detection of health conditions. Early detection should integrate monitoring, compensation, and prevention to provide a value beyond the assessment to address clinical (e.g. infrequent, delayed and limited focus of assessment) and individual (e.g. privacy concerns, complex terminology, self-monitoring devices constraints, fear to diagnosis of diseases) barriers [28]. In addition, tertiary prevention is exemplified by monitoring how often and how well instrumental activities of daily living are performed by aging adults [21].

However, these technologies might not be enough to support preventive health self-monitoring as this might require: 1) a better 
understanding of preventive self-monitoring needs [25], and 2) a proactive activation of people's self-care abilities [13, 35] for prevention to complement the role of the "informed, activated patient" [35]. Based on the related work, we investigated challenges that older adults can experience in day-to-day preventive self-monitoring.

\section{USER STUDIES}

The study integrated qualitative studies and a quantitative survey across the following three main steps. First, an initial and exploratory phase to define a relevant target group was conducted using interviews and cultural probes. Second, we selected a specific health parameter of interest (i.e. blood pressure) for our study. We conducted several workshops, semi-structured interviews and blood pressure self-measurement activities in people's homes. Finally, the third step was based on a quantitative study for data verification and further exploration. These three main steps are described in the following subsections.

\section{Defining our target group}

An initial qualitative study took place including ten participants (four women, six men) with an average age of 61.8 years (SD 11.95). Since chronological age was not a central issue, participants were selected according to their relation to the Danish healthcare system matching the three levels of preventive health measures (i.e. primary, secondary and tertiary prevention [33]). Four participants were categorized in the primary prevention group (one healthy without diagnosis and three taking preventive medication and in risk of getting into treatment), three participants were categorized to be in the secondary prevention group (people with chronic diseases that have changed their lifestyle to prevent further deterioration due to the illness), and three participants in the tertiary prevention group (two people in physical therapy after arterial thrombosis and one previous cancer patient). Participants were interviewed regarding their motivation in relation to the knowledge they had of their current health status, their health data sharing needs, what motivates them to change behavior, their use of self-monitoring technologies at home, and their experience regarding self-monitoring technology being perceived as beneficial or not. Additional material (photos taken by the participants using disposable cameras) was collected as probes.

\section{Exploring challenges}

Based on knowledge from the previous step, we decided to focus on people that are motivated to maintain an existing healthy condition and avoid the decay or the onset of illness, i.e. prevent fragility. We then recruited a new group of participants from a local activity center. The participants were ten active older adults and perceived themselves to be rather 'healthy'. They were between 65 and 84 years old. At the activity center our work extended into a user-involved design process including interviews and workshops together with the older adults. We investigated challenges and attitudes towards the preventive action of blood pressure self-monitoring. We selected BP for analysis of selfmonitoring due to its complexity (number of instructions to follow in order to perform a valid measurement), importance (relationship with other risk factors in the Danish context), and its availability (e.g. presence of blood pressure devices at older adult's homes) identified through the initial photography probes.
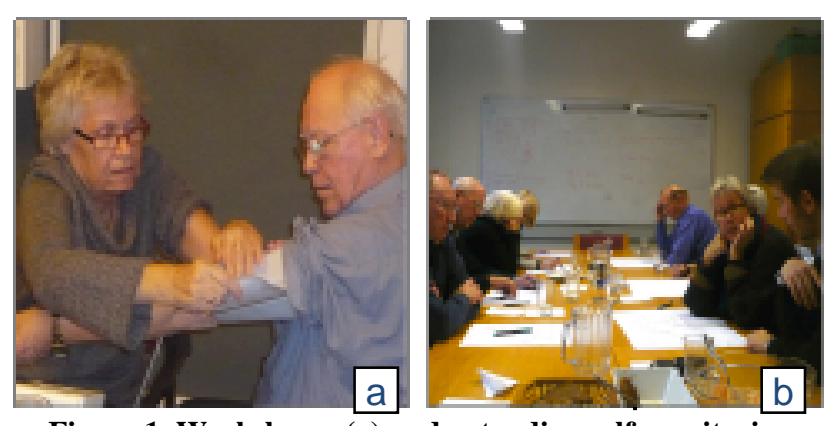

Figure 1. Workshops: (a) understanding self-monitoring activities; (b) Understanding older adults' routines.

In addition, a nurse was engaged in the project and participated in the first workshop. Two of the participants left the project after the first workshop due to the detection of high BP. All sessions were recorded and took place at the activity center. In the following subsections, we will describe the workshop activities followed by their results in the next section.

\section{Understanding self-monitoring activities}

An early goal for the project team was to get a better understanding of self-monitoring activities and its importance regarding older adult's own health status. The workshop participants received an introduction to BP (causes, symptoms, consequences, actions to control $\mathrm{BP}$ ) and how to measure it by the nurse affiliated with the project. During the workshop participants were asked to take their own BP (see Figure 1a) and they all engaged in the activity using a digital BP device. A group discussion was performed during and after each activity. Findings from the first workshop indicated that the complexity of the BP rules challenged self-monitoring activities.

\section{Understanding older adults' routines}

A second workshop was conducted after two weeks. We employed a modified version of the Day Reconstruction Method (DRM) [17] to further investigated older adults' daily routines. We applied our modified DRM to explore difficulties and possibilities in integrating BP measurement activities in everyday routines.

As part of the workshop, participants engaged in a drawing and

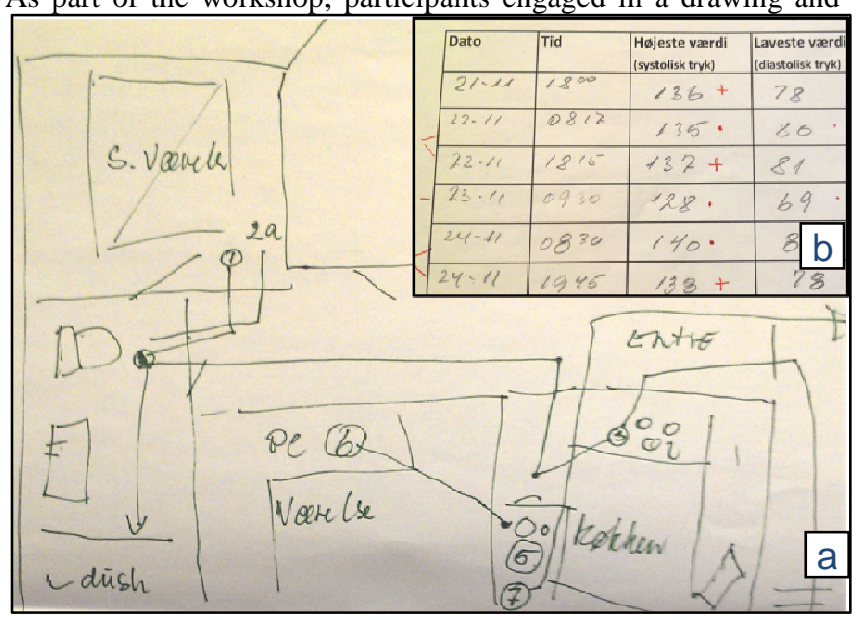

Figure 2. (a) A sketch from the home; (b) a paper-based template to register the BP measurements at home. 
reflection activity regarding their homes and daily routines (see Figure 1b). In detail, the workshop participants were asked to: a) draw a sketch of their homes (see an example in Figure 2a), b) list their activities before they leave home (in the morning or when they consider their morning to be concluded) and after they arrive home (in the afternoon, or when they perceive that they started their 'evening' activities), c) order the activities using numbers on the drawing. One researcher gave an example of how to draw the sketch of the home and how to list the activities before the drawing activity started. One senior needed assistance from a researcher to conduct the task. After the activity, participants shared their sketches and routines. This was followed by a group discussion about the possibilities and implications of using selfmonitoring technologies across their daily activities
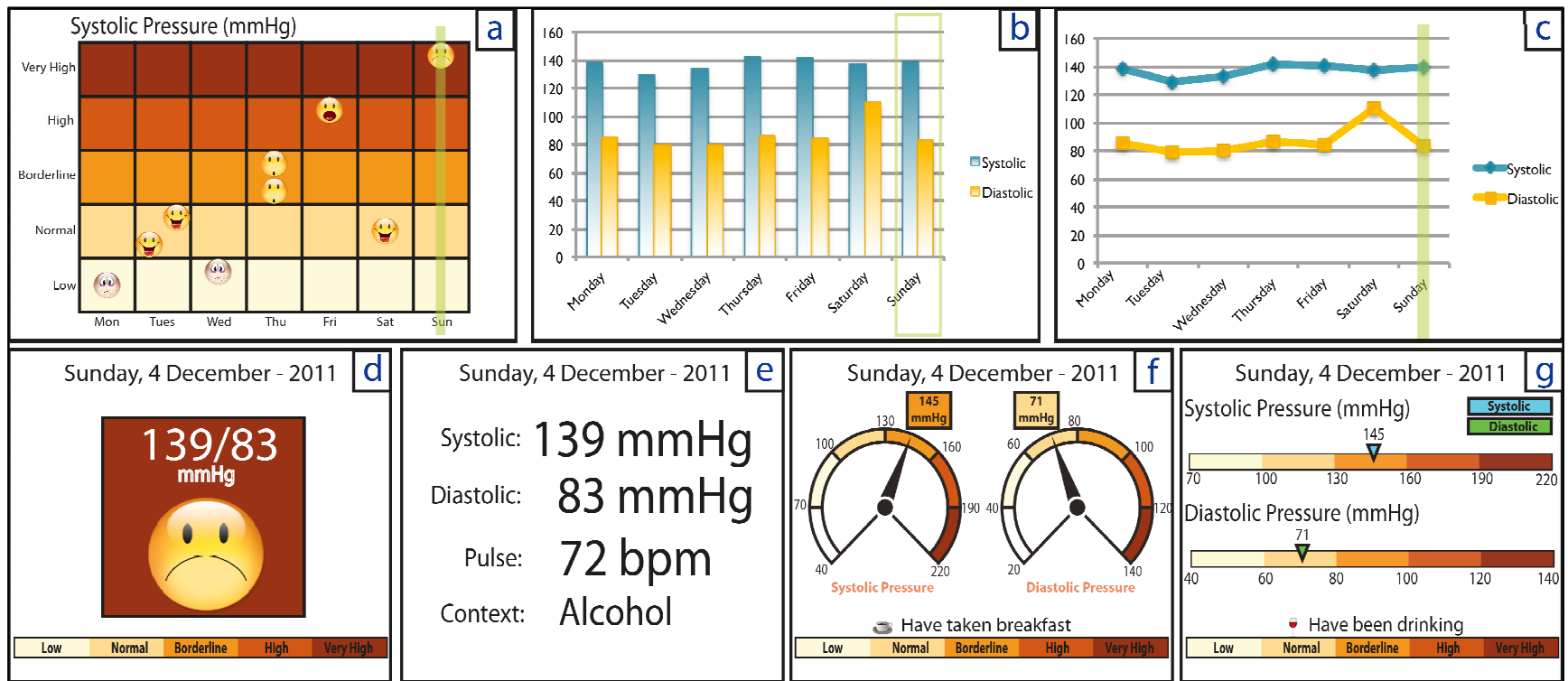

Figure 3. Examples of visualizations using: (a) icons for overview; (b) bar graphs for overview; (c) line graphs for overview; (d) icons for details; (e) text for details; (f) speedometer for details; and (g) bars with encoded colors.

\section{Performing a self-measurement task}

Findings from the second workshop revealed differences between older adults' morning and evening routines. In general, the evening routines were less homogeneous and stable due to an active lifestyle. Therefore, we further investigated how older adults could perform self-monitoring activities at home and the participants were requested to perform BP self-measurements until the next workshop.

The participants were provided digital BP devices and were asked to measure and register their own $\mathrm{BP}$ for at least three days (mornings and evenings) in a row during a one week period (as suggested by the nurse and according to existing guidelines on how to perform a correct BP measurement at home) [30]. These guidelines (e.g. do not smoke, drink coffee, eat or consume alcohol half hour before the measurement) were given verbally and on a paper to the participants. The participants also received a paper-based template where to register and log their BP values. The template included fields such as date, time, higher value (systolic pressure), lower value (diastolic pressure), pulse and a space for additional comments i.e. which arm they used to take the BP (see Figure 2b).

\section{Validation and Interpretation of $B P$ values}

To share their experiences regarding self-monitoring and to validate the previous findings, the workshop participants were invited to a third workshop. At the workshop, the participants presented the collected BP information, the BP rules they had applied and difficulties during the task.
Next, we investigated what and how BP information can be presented to older adults in an understandable way. The participants were given eight different color image-pairs, combining weekly and daily measurement overviews to elicit feedback regarding visualizing data from self-monitoring technologies as suggested by [3]. Examples of these weekly and daily overviews are illustrated in Figure 3. For example, the weekly BP overviews were represented in three different formats using: 1) encoded charts with icons, circles, starts and squares without specifying an exact value (see Figure 3a); 2) vertical and horizontal bars (see Figure 3b); and 3) line charts (see Figure 3c).

Furthermore, the daily overviews were represented by four different formats using: 1) icons to represent a specific BP values (see Figure 3d); 2) text values of BP (see Figure 3e); 3) speedometers visualizing $\mathrm{BP}$ values (see Figure $3 \mathrm{f}$ ); and 4) encoded charts using BP values (see Figure $3 \mathrm{~g}$ ). These weekly and daily visualizations were combined and one pair was shown at the time. The participants commented and gave feedback on the presented visualizations. Finally, the participants engaged in a group discussion about how to visualize and facilitate interpretation of health data for citizens that have no previous knowledge of medical data.

\section{Quantitative study}

The workshops provided a deeper understanding of the challenges related to self-monitoring activities, especially for independently living older adults that like to prevent fragility. However, we wanted to include a broader population to further explore selfmonitoring activities and the workshop results. We developed a 
quantitative study (a Danish web-survey) that touched upon the same questions as the initial qualitative study. The survey also included questions regarding current health status (e.g. any chronic disease) and personal information (e.g. age, gender, location). We received 191 responses to the survey, but only 165 were completed and considered in our analysis. Participants were adults between $22-83$ years old.

\section{CHALLENGES OF PREVENTIVE SELF-MONITORING}

In our quantitative survey, 131 respondents (out of 165) do not have any chronic disease and 86 of these participants own a health self-monitoring device. This indicates that people without any chronic condition are interested in their own health, keeping a desire 'to stay young' as long as possible. In the study, nine themes emerged from a combination of the most relevant topics and discussions sprung out of from the workshops and other user activities (inductive) and the most relevant issues regarding selfmonitoring (e.g. lack of contextual information, privacy issues, difficulties understanding clinical terminology, attitudes and perceptions of people about self-monitoring, and problems with measuring devices $[1,28,30,34])$ from the literature (deductive). The nine themes related to home-based self-monitoring were: 1) rule complexity, 2) reliability of the measurements, 3) interpretation, understanding and health awareness, 4) the sharing activity for prevention, 5) motivational sources, 6) the role of the doctor for prevention, 7) the use of self-monitoring technology, 8) the home as a distributed information space, and 9) visualization of BP values. We now present the nine challenges that emerged from our study and how to support a successful integration of preventive BP self-monitoring in everyday life.

\section{Rule complexity}

The first challenge for preventive $\mathrm{BP}$ self-monitoring is rule complexity. Rule complexity relates to the rules a person must adhere to guarantee a valid (BP) measurement. For example should one sit and rest for 5 minutes before taking the BP. Our workshop participants mentioned several difficulties in measuring $\mathrm{BP}$ in accordance with the established rules. A challenge was that they did not (perceive to) have enough time to correctly take a measurement (and hence apply to the rules of rest before a BP measurement or other time-constraining rules such as not eating, drinking or smoking 30 minutes before the measurement). Indeed, older adults may have other activities than self-monitoring to attend during the day.

When examining the self-documented data from the monitoring activity at home, it was clear that all participants performed their morning BP measurements. However, some participants experienced difficulties integrating the evening measurements into their lives and hence omitted the evening measurements. Thus, developing and adjusting routines to suit the $\mathrm{BP}$ rules was complicated during the evening due to for example an active lifestyle. Indeed, a male participant with a very active social live could not find the time to carry out the preventive measurement task for three days (2 measurements a day) in a row (this configuration was suggested by the project nurse). He said "I cannot do it this week [looking at his agenda], maybe next week I can try...". Analyzing the drawings the workshop participants made of their homes and the herein situated activities confirmed the characteristics of morning and evening measurements. We found in general that the morning routines were more established than the evening routines, as they appeared more stable and were easy to recall for the participants.

\section{Reliability of the measurements}

This challenge concerns the reliability of measurements taken by laypeople in their homes. Due to for example rule-complexity in conducting valid home-based measurements and the lack of contextual information during the measurements, the reliability of the measures can be compromised. For instance, the participants had to take more than one BP measurement to assure and verify their measurements. Four of the participants performed the measurement two or three times in a row on either one or both arms (this being contextual information). Even though we asked them to write down such contextual information (e.g. if they have rested, talked or smoked before or during the measurement) that can indicate non-adherence to the rules, the only information they wrote down was about which arm the measurement was from. When the contextual information is missing or misleading, readings can show an erroneous value, without being explained why, that might not reflect the current condition. For example, the readings might show hypertension stage one, but this could be related to missing information (e.g. the participant did not follow the guidelines and did not report the rule non-adherence) around the measurement. We also observed that measurements could be unintentionally done in a non-correct fashion, for example due to a misplaced cuff on the arm. We additional found possible misreporting due to handwriting mistakes or a non-functional device.

\section{Interpretation, understanding and health awareness}

People should not only become aware and understand the consequences of unintentional mistakes or misreporting that can compromise the reliability of the measurements. They should be able to interpret and understand the meaning and consequences of the measurements. From the initial qualitative study, a participant said "the weight scale shows too much. Could my weight be a side effect to the pills? I can feel and see that I have gained weight". From our workshop activities it became clear that the participants were well aware of, and understood, the importance of lowering their BP if they were measured with a high BP. However, how to lower the BP was less understood and the participants were interested in knowing more about how they could decrease their $\mathrm{BP}$ value.

In general, participants could use the BP measuring device provided by us after a limited initial training. However, moving from a correct device usage to a proper understanding of the measurements (i.e. the numeric values of the BP) was more challenging. While the participants managed to take a proper measurement, they failed to understand, or interpret, the acquired values. For instance, half of the participants had their BP value over the normal level when they did their first measurement and hence the nurse asked them to repeat the measurement on the opposite arm to compare the BP values. The nurse suggested that the lower value (taken from either of the two arms) should be considered as the reference point for future measurements. The nurse stated that a higher value in a specific arm is due to an obstruction of the arteries in the side of that arm. The nurse played an important role explaining the meaning of the values in each arm and the importance of taking the measure at least twice a day (morning and evening) three days in a row.

When reporting the BP measurements using the provided paperbased templates, participants use symbols (e.g. lines and '+'signs) to group them and identify values within the normal BP range. The participants also took additional readings from both 
arms to compare and understand the BP values and hence support the interpretation. Furthermore, an understanding of their current health situation can support older adults in getting a sense of health awareness towards maintaining or achieving a better health status. A participant from our qualitative study stated "For many years I forgot about exercise. 5-6 years ago I had a case of slipped discs. I have avoided surgery with the help of painkillers and exercise". Thus, an interpretation and understanding of the current health situation can help older adults to achieve better health outcomes. In general, we confirmed that high blood pressure is indeed a non-communicable disease as people may not be aware that they have a high BP. As previously stated, two participants left the project after becoming aware of their high BP.

\section{The sharing activity for prevention}

The workshop participants all had concerns about sharing illnessrelated information and performing measurement-activities outside the household. It was for example not acceptable to perform BP measurements at a friend's house or at a restaurant, not even if they went to a more private space such as the restroom. It should be noted that the measurements were related to preventive monitoring, rather than monitoring of an identified illness. This can have, as a minimum, two effects: 1) the older adults did not feel that it was a matter of "life or death" if they did or did not perform the BP measurement, and 2) they did not like to deal with health-related issues (preventive care) if their activities (planned or not) could be miss-understood as illnessrelated. This was confirmed by the qualitative study. For example did one participant state "I' $m$ not interested in coming off as being sick, because I'm not". In general the respondents from the quantitative study were not willing to share their own health information and preferred to not involve others in their health management. However, $63 \%$ of participants mentioned that they would like to share their data with their physicians if needed. In contrast, some participants from the qualitative study shared the interpretation task with someone from the immediate family, for example a spouse, to add a confirmation step and see if the measurement was correctly taken.

\section{Motivational sources}

When the workshop participants understood the importance of BP measurements, they further asked questions and showed more interest in our activities. In the project, we identified three main motivational sources. The first one came from the individuals and their feelings of independence. A participant from the qualitative study expressed "To have to be dependent on others\#\#I don't like that. I would rather be able to do it myself." In particular, the older adults in the qualitative study expressed major concerns regarding the feeling of being independent. A participant said, "It is hard to have to depend on others, and to continue to do so".

The second motivational source derives from social relationships and a general interest in health. For instance, the qualitative study participants mentioned reasons for why to engage in preventive self-monitoring. A female participant said that (she and her companion) would like to continue to be able to take care of their grandchildren, love each other and live their lives. In the quantitative study, 42 participants had acquired a self-monitoring device due to a general interest in their own health.

The third motivational source came from the performance of daily activities. Participants expressed a desire to continue being able to do the things they normally do and remain in their homes as long as possible. For instance, a participant from the qualitative study expressed "Going out and shoveling snow\#\#\# was surprised by how well it went. Our house is in a corner area, so there was a lot of snow. But I wanted to do it! I am a little stubborn. I have to continue to be able to do the things I have always done". This was confirmed in the quantitative study where 43 participants (out of the 110 respondents that owned a self-monitoring device) use monitoring devices for prevention as they would like to remain 'as young as possible' and monitoring their health can help them to achieve that.

\section{The role of the doctor for prevention}

The quantitative study shows that $75 \%$ of the participants that use self-monitoring devices for prevention would like to use them as a supplement of the existing health services (e.g. counseling, doctor visits, etc.). But only $60 \%$ would use them as a substitute. Older adults are more reluctant to replace primary healthcare with selfmonitoring technology. However, the study shows that older adults are willing to acquire new self-monitoring technologies to maintain or improve their health, but only after the technology has been out on the market ( $22 \%$ of the older respondents) for a while and been tested on beforehand (30\% of the older respondents).

During preventive care activities, the role of the doctor can take a more advisory function. The study showed that participants did not consider involving the doctor unless they are really sick or their measured values are not within the normal range. Here, doctors can help in the interpretation of the measured values or support and follow up on people that perform preventive care activities. Such activities might support people to get selfconfident with preventive self-care activities. A participant from the qualitative study expressed "The last two years we have been able to get health checked through the company. But I always knew approximately how my health was, but it is always nice to be confirmed in your believes and get a self-esteem boost. It also shows that the way I chose to live ... that I don't really need to make any changes. By getting several checks you can also see a trend, which tells me where I'm heading. That is a good indication".

\section{The use of self-monitoring technology}

The workshop participants had a rather general understanding of technology. They all had mobile phones and modern household appliances. They were to some extent interested in computers, as they all followed a beginner's computer class at the activity center where we arranged the workshops. The participants (except from one, that later withdraw from the project) did not have previous experience using a BP measurement device at home. However, they could all use the digital, off-the-shelf BP measurement device after its introduction.

From the quantitative studies, we could see that health-monitoring technologies have presence in people's homes. Heart rate, BP monitors and pedometers were the most frequently available devices among the 131 respondents without chronic conditions. Furthermore, they would prefer to automatically measure: 1) physiological parameters such as weigh and blood pressure (89), 2) their diet (74), 3) wellbeing parameters such as depression, stress, etc. (44), 4) cognitive functions such as memory, analytical skills, etc. (43). 24 participants replied that they would not like to measure any health data about themselves.

Regarding older adults, participants would prefer to automatically measure physiological parameters (47), diet (24), wellbeing (18), 
cognitive functions (21) and 12 older participants replied that they would not like to measure any health data about themselves.

In addition, BP devices were also found in people's homes during the initial qualitative study through the collection of cultural probes. This confirmed our selection of BP as the main health parameter for this study.

\section{The home as a distributed information space}

When the project participants draw sketches of their homes, it was possible to observe how the older adults visualized and communicated their routines. After analyzing the drawings, four similarities emerged: 1) there are common places for specific routines among the participants (e.g. brushing the teeth or hair happens in the bathroom), 2) morning routines are more established than evening routines (as explained above), 3) activities can occur in one or more places (e.g. reading the newspaper), and 4) more than one activity can take place at the same time (e.g. eating breakfast and reading the newspaper).

\section{Visualization of BP values}

Our workshops revealed that older adults have some difficulties understanding BP readings. Even though they could perform the measurements, they experienced difficulties interpreting the resulting values (as described above). Thus, we explored different ways to enhance their understanding of BP data using mockups visualizations.

In this activity, we explored two levels of information, the weekly overview and the detailed view of measured values from the current day as illustrated in Figure 3.

\section{Weekly overview}

First, participants found the visual encoding of the charts useful. They used it as an indicator of the meaning of the different BP values such as low, normal, borderline, high, and very high (see Figure 3a). A workshop participant expressed "it is simple and easy to understand...". However, they felt that the charts were not precise when the BP values are not shown and they would like a combination with the line charts (see Figure 3c).

Second, participants found the simplicity of the line chart useful. However, some participants did not find the dots in the curve as relevant. For example, one participant commented, "... maybe you can add the smile-faces on the curve". This presupposes that people might need to know the different meaning of the smilefaces if they are associated to the home-based measurements.

Third, participants agreed about a simple way to see the actual value of the measurement through the bar graphs (see Figure $3 b$ ). However, they did not understand the meaning of the values and suggested that the pillars could relate or have indicators or some mechanisms to present the meaning of the value.

\section{Daily details}

First, participants found the icons useful but also indicated some problems with the iconic data representation (see Figure 3d). A workshop participant expressed "It is simple, appealing and funny, and it is easy to understand but not precise".

Second, participants relied on text values as they allowed them to simply extract and see the actual measurement-value (see Figure $3 \mathrm{e})$.
Third, some found the speedometer useful (see Figure 3f) saying that it is 'kind of' ok since the blood pressure is like a speedometer. One participant said "it [blood pressure] goes up and down". However, they also agreed that in some cases a speedometer might not be precise.

Finally the participants found the color indicators of the encoded bars - "red-toned bars" useful and perceived them as a good strategy to show the daily measurements. A participant said, "The idea of the daily overview is kind of ok" (see Figure 3g).

\section{DESIGN OPPORTUNITIES FOR PREVENTIVE BLOOD PRESSURE SELF-MONITORING}

The findings indicate that current (BP) self-monitoring technology cannot fully support the needs of preventive self-monitoring in practice. The complexity of the rules that should be followed to guarantee valid BP measurements and the lack of contextual information may for example compromise the reliability of the measurements. Even though participants were able to take the (morning) measurements in a complex distributed information space such as the home, they failed to interpret and understand their measurements.

In this section, we discuss design opportunities for home-based self-monitoring that aim to enhance people's abilities for self-care and promote preventive self-monitoring activities at home.

\section{Rethinking preventive self-monitoring of health parameters towards a positive experience}

Most monitoring devices have been designed taking the clinician's perspectives into account to help people, including older adults, to monitor health parameters to gain control and/or prevent illness and disabilities [28]. However, current monitoring devices have, to a large degree, neglected personal perceptions and the motivation that make people control their health and wellbeing. Exploring the social aspects of people's everyday life can increase motivation to perform self-monitoring (e.g. RunWithUs [12]). As described earlier, there are different motivational sources that people have that go beyond the actual self-measuring activity. Older adults want to be independent as long as possible and continue with the activities they are used to, stay longer in their own place, etc. The social environment is really important, as older adults want to stay active and be able to visit friends, attend the activity center, travel or take care of their grandchildren. To not consider older adults' motivational factors might negatively influence the use of preventive self-monitoring in everyday life. Most of the participants considered preventive self-measurements as an individual activity that should be performed at home and not in a social environment. The participants feared they might be perceived as ill when they are not.

Furthermore, the complexity of the rules associated with biomeasurements turns the preventive self-measurement tasks into a complex endeavor that interfere with everyday activities. Hence, considering the peculiar characteristics of preventive selfmonitoring, there is a need to provide older adults and society in general with a positive experience regarding self-measurement activities. Creating a positive experience may imply rethinking preventive self-monitoring as a tool that can allow people to achieve their personal goals driven by their own motivation. A positive experience associated with the use of preventive selfmonitoring technology can facilitate a better integration with older adults' daily routines. This integration might facilitate older 
adults' participation in self-care activities and should be considered from the beginning of the design process [14]. Indeed, as Bannon [2] proposed more than 20 years ago, we should consider humans as actors and not factors.

In society, a positive experience might increase the sharing of health information with close family members and friends as monitoring can be seen, for example, as a wellness activity thus avoiding stigmatization.

\section{Supporting the active and informed citizen}

In order to support the active and informed citizen while performing BP self-monitoring at home, healthcare technology designers should (re) consider the role of older adults as proactive people [32]. Furthermore, a proper understanding of why people follow a health intervention (e.g. using prescribed medication or doing self-monitoring) is needed not only to enhance the individual health awareness but also to facilitate its integration in people's everyday life [7]. As stated in this paper, older adults want to interpret, understand and get meaning from the measurements in relation to their current health status. Thus, selfmonitoring can support this learning activity [8]. In doing so, the role of the doctor is shaped to support this interpretation process (as preferred for some of our participants) or to confirm their personal interpretation and understanding of the current health situation. To facilitate the interpretation and understanding of health data, visualization techniques can be used to provide older adults with the needed information at hand to improve health awareness [22]. For instance, our visualization activity reported that older adults rely on text values rather than icons. They want to know the exact values even if they do not understand their meaning. The workshop participants valued the visualizations as they gave both an overview and details of BP data (see Figure 3g). By showing a combination of charts and a color scheme representing BP levels (low, normal, borderline, high, very high) visualizations complement and enrich the actual $\mathrm{BP}$ value on the monitoring device. A graphical interface that combine encoded and line charts (as suggested by participants) can provide a clear overview embedding temporal dimension (logging and sense making of BP data) to support older adults self-reflection processes.

Furthermore, the lack of contextual information inherent to the monitoring devices provides opportunities for Ubicomp and context-aware technologies to support self-monitoring practices of health data in and out of a complex distributed information space not only for chronic care [15] but also for preventive care. Selfmonitoring technology for preventive care should provide the opportunity for older (and younger) adults to enter into a reflection process, regarding their health data and help them to take action on their own (current and future) health situation. Existing efforts on guidelines for data collection towards supporting self-reflection [24] of personal information are not yet reflected or available in self-monitoring devices at older adult's homes. We suggest that a simple tagging functionality of contextual information (e.g. whether the measurement was taken before or after the breakfast or if the person has been drinking alcohol or coffee before the measurement) can help older adults to reflect and understand the dynamic nature of their measurements. The tagging functionality can also serve as a tool for validation of the reliability of the measured data and to enhance the user's health awareness of their current health situation. Thus, using visualization and tagging techniques can facilitate the interpretation of health data and support the active informed citizen to learn about their own health.

All these aforementioned opportunities can support older adults' health education and self-learning activities regarding preventive care.

\section{CONCLUSION}

In this paper, we have explored preventive self-monitoring activities in non-clinical settings for older adults. The study has focused primarily on BP measurements, but some of the herein presented findings may be applicable to other bio-monitoring scenarios. However, this should be further studied and understood as people might use diverse self-monitoring technologies for prevention.

Successful home-based, preventive health monitoring can be highly beneficial for both the individual and society. However, our study reveals a number of challenges that can lower the positive impact of these measurements, or even making them impossible. An example challenge is how to achieve a successful integration of the guidelines into an individual's daily routines. If a successful integration can be made, opportunities such as a better understanding of what life-situations affects the body both positively and negatively can be obtained.

To support a successful integration of health self-monitoring (e.g. blood pressure) into older adults everyday lives one have to consider the individual motivational factors, the home as a distributed information space, and how health information are presented. For our participants, a precise representation of the BPvalues was of outmost importance to facilitate the interpretation and understanding of ones' health.

The findings from our study call for rethinking preventive selfmonitoring of BP (and possibly other health parameters) to generate a positive user-experience by considering older adults' perspectives, needs and desires. One way to provide this positive experience is by supporting the active and informed citizen in order to improve older adults' health awareness while supporting their independence at home. These opportunities may be transferrable to other age groups, as more people are becoming aware and starting preventive care activities before they get older.

\section{ACKNOWLEDGMENTS}

We would like to thank all professionals and participants involved in the Lev Vel Consortium for their cooperation. Lev Vel is funded by The Danish Council for Technology and Innovation and The Capital Region of Denmark.

\section{REFERENCES}

[1] Ashiful, A., Zarifi Eslami, M., and Sikkel, K. Elderly and homecare tasks: A literature review on problems. In Proceedings of the $4^{\text {th }}$ IADIS International Conference eHealth 2012, IADIS Press. 211-216.

[2] Bannon, L. From human factors to human actors: The role of psychology and human-computer interaction studies in system design. Design at work: Cooperative design of computer systems, 1991, Lawrence Erlbaum Associates, Inc.: Hillsdale, New Jersey, USA: p. 25-44.

[3] Beaudin, J.S., Intille, S.S., and Morris, M.E. To track or not to track: user reactions to concepts in longitudinal health 
monitoring. Jounal of medical Internet research, 2006. 8(4):e29.

[4] Codagnone, C. Reconstructing the whole: present and future of Personal Health Systems. Publication Office of the European Union, 2009.

[5] Coley, C., et al., Preferences for home vs hospital care among low-risk patients with community-acquired pneumonia. Archives of Internal Medicine, 1996, 156(14): p. 1565 .

[6] Czeresnia, D., The concept of health and the difference between prevention and promotion. Cadernos de Saúde Pública, 1999. 15(4): p. 701-709.

[7] Dalgaard, L.G., Grönvall, E., and Verdezoto, N., Accounting for Medication Particularities: Designing for Everyday Medication Management. In Proc. PervasiveHealth 2013. IEEE (2013), 137,144.

[8] Dunlosky, J. et al., Do self-monitoring interventions improve older adult learning? J Gerontol B Psychol Sci Soc Sci, 2007. 62(Special Issue 1): p. 70-76.

[9] Fahmi, P.N.A., Viet, V., and Deok-Jai, C. Semi-supervised fall detection algorithm using fall indicators in smartphone. In Proc. ICUIMC 2012. ACM, New York, NY, USA, p. 122.

[10] Fisk, A.D., et al. Designing for older adults: Principles and Creative Human Factors Approaches. Vol. 2. 2009.

[11] Gesano, G., Heins, F., and Naldini, A. Regional Challenges in the perspective of 2020, Regional disparities and future challenges. Background paper on Demographic Challenge. ISMERI Europa 2009. Available from: http://ec.europa.eu/regional_policy/sources/docgener/studies/ pdf/challenges2020/regional_challenges_demographic_chall enge.pdf

[12] Gil-Castiñeira, F., et al., RunWithUs: a social sports application in the ubiquitous Oulu environment, In Proc. MUM 2011, ACM, New York, NY, USA, 195-204.

[13] Glasgow, R.E., et al., Does the chronic care model serve also as a template for improving prevention? Milbank Quarterly, 2001. 79(4): p. 579-612.

[14] Grönvall, E. and Kyng, M. On participatory design of homebased healthcare. $C T \& W, 2012:$ p. 1-13.

[15] Hayes, G.R., et al., Opportunities for pervasive computing in chronic cancer care, In Pervasive Computing. Springer Berlin Heidelberg, 2008. 262-279.

[16] Juel, K., Sørensen, J., and Brønnum-Hansen, H. Supplement: Risk factors and public health in Denmark. Scandinavian Journal of Public Health, 2008. 36(1 suppl): p. 1-227.

[17] Kahneman, D., et al. A Survey Method for Characterizing Daily Life Experience: The Day Reconstruction Method. Science, 2004. 306(5702): p. 1776\#\#\#1780.

[18] Kane, R.L., Kane, R.A., and Arnold, S.B. Prevention and the elderly: risk factors. Health Services Research, 1985. 19(6 Pt 2): p. 945 .

[19] Kaufman, S.R., Construction and practice of medical responsibility: Dilemmas and narratives from geriatrics. Culture, Medicine and Psychiatry, 1997. 21(1): p. 1-26.
[20] Klasnja, P., Consolvo, S., and Pratt, W. How to evaluate technologies for health behavior change in HCI research. In Proc. CHI 2011. ACM, New York, NY, USA, 3063\#\#\#3072.

[21] Lee, M.L. and Dey, A.K. Embedded assessment of aging adults: A concept validation with stakeholders. In Proc. PervasiveHealth 2010. IEEE (2010).

[22] Lee, M.L. and Dey, A.K. Reflecting on pills and phone use: supporting awareness of functional abilities for older adults. In Proc. CHI 2011, ACM, New York, NY, USA, 2095 2104.

[23] LEVVEL. Forside\#\#\#LEVVEL. 2011. Available from: http://www.lvvl.dk/.

[24] Li, I., Dey, A.K., and Forlizzi, J. Understanding my data, myself: supporting self-reflection with ubicomp technologies. In Proc. UbiComp 2011, ACM, New York, NY, USA, 405414.

[25] Lupton, D., M-health and health promotion: The digital cyborg and surveillance society. Soc Theory Health, 2012. 10(3): p. 229-244.

[26] Mallery, L. and Rockwood, K. Preventive Care for the Elderly: Uncovering the unmet needs of this population. Canadian Family Physician, 1992. 38: p. 2371.

[27] Mik-Meyer, N. and Villadsen, K., Magtens former: Sociologiske blikke på statens mфde med borgeren. Sociologiske perspektiver på statens møde med borgeren 2007, Copenhagen: Hans Reitzels Forlag.

[28] Morris, M., Intille, S., and Beaudin, J. Embedded assessment: Overcoming barriers to early detection with pervasive computing. In Pervasive Computing. Springer Berlin Heidelberg, 2005. 333-346.

[29] O'Brien, E., et al., European Society of Hypertension recommendations for conventional, ambulatory and home blood pressure measurement. Journal of hypertension, 2003. 21(5): p. 821.

[30] Parati, G., et al. European Society of Hypertension Practice Guidelines for home blood pressure monitoring. Journal of human hypertension, 2010. 24(12): p. 779-785.

[31] Rockwood, K., et al., Frailty in elderly people: an evolving concept. CMAJ: Canadian Medical Association Journal, 1994. 150(4): p. 489\#\#\#495.

[32] Rogers, Y., Moving on from Weiser's Vision of Calm Computing: Engaging UbiComp Experiences, In Proc. UbiComp 2006: Ubiquitous Computing, P. Dourish and A. Friday, Editors. 2006, Springer Berlin Heidelberg. p. 404421.

[33] Stults, B.M., Preventive health care for the elderly. Western Journal of Medicine, 1984. 141(6): p. 832.

[34] Tentori, M., Hayes, G.R., and Reddy, M. Pervasive Computing for Hospital, Chronic, and Preventive Care. Found. Trends Hum.-Comput. Interact., 2012. 5(1): p. 1-95.

[35] Wagner, E.H., Chronic disease management: what will it take to improve care for chronic illness?. Effective clinical practice: $E C P, 1998$. 1(1): p. 2-4.

[36] Wei, J.Y., Age and the Cardiovascular System. New England Journal of Medicine, 1992. 327(24): p. 1735-1739. 
[37] WHO, Adherence to long-term therapies, Evidence for action, 2003. World Health Organization.

[38] WHO. 10 Facts on Ageing and the Life Course. 2012 [cited 2012 May]. Available from: http://www.who.int/features/factfiles/ageing/en/.
[39] Wild, K., et al., Unobtrusive in-home monitoring of cognitive and physical health: Reactions and perceptions of older adults. Journal of Applied Gerontology, 2008. 27(2), $181-200$ 